\title{
Low-Temperature Scanning Tunneling Spectroscopy of Semiconductor Surfaces
}

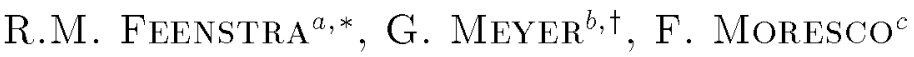 \\ AND K.H. RIEDER ${ }^{c}$ \\ ${ }^{a}$ Department of Physics, Carnegie Mellon University \\ Pittsburgh, Pennsylvania 15213, USA \\ ${ }^{b}$ Paul Drude Institut für Festkörperelektronik \\ Hausvogteiplatz 5-7, 10117 Berlin, Germany \\ ${ }^{c}$ Institut für Experimentalphysik, Freie Universität Berlin \\ Arnimallee 14, 14195 Berlin, Germany
}

\begin{abstract}
Low-temperature scanning tunneling spectroscopy measurements on semiconductor surface are described. We consider both surfaces which do not possess surface states within the bulk bandgap, such as GaAs(110), and surfaces which do have states within the gap, such as $\mathrm{Ge}(111) 2 \times 1$ and $\mathrm{Ge}(111) \mathrm{c}(2 \times 8)$. Band bending in the semiconductor due to the electric field in the vacuum penetrating the semiconductor is found to be a substantial effect in the former case. Transport limitations in the semiconductor give rise to additional voltage drops, which can be observed by making measurements over a wide range of tunnel current magnitudes.
\end{abstract}

PACS numbers: 73.20.Hb, 71.20.Nr, 68.37.Ef

\section{Introduction}

Studies of semiconductor surfaces using scanning tunneling spectroscopy (STS) have been actively pursued for nearly 20 years, but it is only over the past 5 years that measurements at low temperatures have been conducted. Most notably, discrete accumulation layer states have been resolved in low-temperature spectra of InAs(110) surfaces [1], and the magnetic field dependence of these states has revealed a wealth of interesting phenomena [2]. GaAs(110) surface have also

* corresponding author; e-mail: feenstra@cmu.edu

${ }^{\dagger}$ present address: IBM Research Division, Zurich Research Laboratory, 8803 Rüschlikon, Switzerland 
been studied at low temperature, including an examination of features associated with dopant atoms near the surface [3-7]. In contrast to these studies of III-V semiconductor surfaces, which do not have electronic states within the semiconductor band gap [8], several low-temperature STS studies have also been reported for surfaces which are dominated by surface states [9-12]. A distinct sharpening of the spectral lines at low temperature is found, making possible a more detailed interpretation of the scanning tunneling microscopy (STM) imaging mechanisms $[9,11]$. Possible temperature-dependent limitations in the tunneling process have also be proposed [12].

In this paper we present results from our recent studies of semiconductor surfaces using low-temperature STS, discussing spectra acquired from GaAs(110), $\mathrm{Ge}(111) 2 \times 1$, and $\mathrm{Ge}(111) \mathrm{c}(2 \times 8)$ surfaces $[7,11,13]$.

\section{Experiment}

All the semiconductor surfaces prepared for our studies have been formed by cleavage of small pieces of semiconductor wafers. For the case of GaAs we start with an $\{100\}$-oriented wafer, and the $\{110\}$ cleavage faces are perpendicular to the wafer surface. For Ge we start with a $\{111\}$-oriented wafers, so that the $\{111\}$ cleavage faces are oriented at $109.5^{\circ}$ to the wafer surface. Our GaAs wafers were $n$-type (Si-doped) with a specified donor concentration of $(2.0-2.6) \times 10^{18} \mathrm{~cm}^{-3}$, corresponding to a room temperature resistivity of about $0.0015 \Omega \mathrm{cm}$. The Ge wafers were $p$-type with room temperature resistivity of $0.2 \Omega \mathrm{cm}$, corresponding to an acceptor concentration of about $2 \times 10^{16} \mathrm{~cm}^{-3}$. All samples were cleaved in ultra-high-vacuum (pressure of about $1 \times 10^{-10}$ Torr). Cleavage was performed at room temperature. For the case of the $\mathrm{Ge}(111) \mathrm{c}(2 \times 8)$ surface the sample was then resistively heated to a temperature of about $500^{\circ} \mathrm{C}$ for a few minutes. Immediately following cleavage and/or heating the samples were cooled to about $50 \mathrm{~K}$ and were introduced into a liquid-He cryostat containing the home-built STM [14]. Results presented in this paper were acquired with the tip and sample at a temperature of about $10 \mathrm{~K}$, unless otherwise specified. Probe-tips were formed prior to sample cleavage by making a controlled mechanical contact of a tungsten tip to a clean copper surface, thereby transferring copper atoms to the end of the tip. Metallic tips are found to reliably form in this manner [7]. Scanning tunneling microscopy images were acquired at a constant current of typically $0.3 \mathrm{nA}$, and at various sample-tip voltage specified below.

Tunneling spectra were acquired using a voltage modulation of $10-20 \mathrm{mV}$ and employing a lock-in amplifier to obtain the conductance. For spectra over a wide voltage range, the technique of continuously varying sample-tip separation was used to ensure a large dynamic range measurements [15], applying an offset to the sample-tip separation of the form $\Delta s=a|V|$, where $V$ is the sample-tip voltage and values of $a$ of typically $1 \AA / V$ were employed. The spectra are normalized by computing the ratio of differential to total conductance, $(\mathrm{d} I / \mathrm{d} V) / \overline{(I / V)}$, 
where some broadening is applied to $(I / V)$ in order to form a suitable normalization quantity (i.e. to avoid divergences which otherwise occur at the band edges) $[15,16]$. For the GaAs normalized spectra shown here we use a broadening of $1.5 \mathrm{~V}$ and for the Ge spectra we use $1.0 \mathrm{~V}$.

\section{Results and discussion}

$$
\text { 3.1. } G a A s(110)
$$

Figure 1 shows a constant-current STM image of an $n$-type GaAs(110) surface, acquired with sample voltage of $+1.5 \mathrm{~V}$ (thus corresponding to electrons tunneling from the probe-tip into normally empty states of the sample). The corrugation of the $1 \times 1$ surface unit cell, measuring $5.65 \times 4.00 \AA$, is clearly visible. In addition, dopant atoms are revealed by the presence of the region of raised corrugation, as labeled by (f)-(j) in Fig. 1. Such dopant atom features in STM images have been extensively studied in the past, at both room temperature (RT) [17-19] and low temperature (LT) [3-6]. Dopant atoms in the surface atomic plane and in typically about 5 subsurface planes are seen. For the present experiment, assuming that dopants as deep as the 5th layer are visible, we obtain a dopant concentration of $5 \times 10^{18} \mathrm{~cm}^{-3}$, in reasonable agreement with the specified donor concentration of our samples. In addition to dopant atoms, we also observe a few other features indicated by $V$ and $R$ in Fig. 1, and we associate these features with either surface defects such as vacancies, or residual surface contamination, respectively.

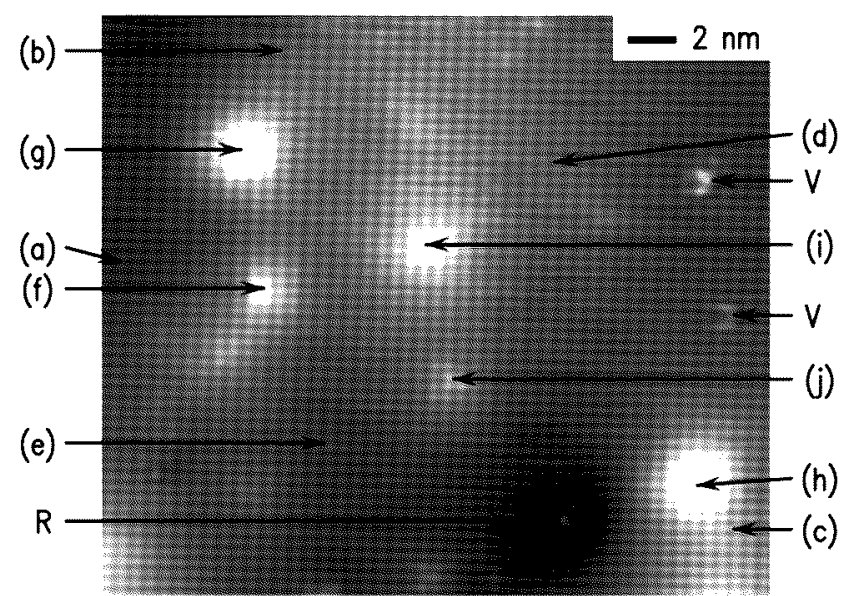

Fig. 1. Constant-current STM image of $n$-type GaAs(110) surface, acquired at a sample voltage of $+1.5 \mathrm{~V}$. The gray scale range is $0.5 \AA$. Locations at which tunneling spectra were acquired are indicated by (a)-(j). Surface defects (probably vacancies) are indicated by $V$ and residual surface contamination by $R$. 
A tunneling spectrum acquired at a typical surface location (not near a defect or dopant atom) is shown in Fig. 2. This spectrum was acquired over a large voltage range using the variable sample-tip separation method described in Sect. 2. The conduction band (CB) and valence band (VB) components in the conductance are clearly seen, and the band edge are marked in the spectrum. We define a band gap as the separation between these band edges, yielding an observed value of $1.60 \pm 0.05 \mathrm{eV}$ for this LT spectrum. This result is slightly larger than the expected LT band gap of GaAs of $1.52 \mathrm{eV}$; this discrepancy may arise from effects of tip-induced band bending [20]. Room temperature spectra actually yield a band gap somewhat closer to the expected value [21], but the band bending effects in that case may be somewhat masked by the expected thermal broadening of the VB and $\mathrm{CB}$ conductance edges into the gap region. One distinct surface-state related feature is observed in Fig. 2 at $+0.72 \mathrm{~V}$.

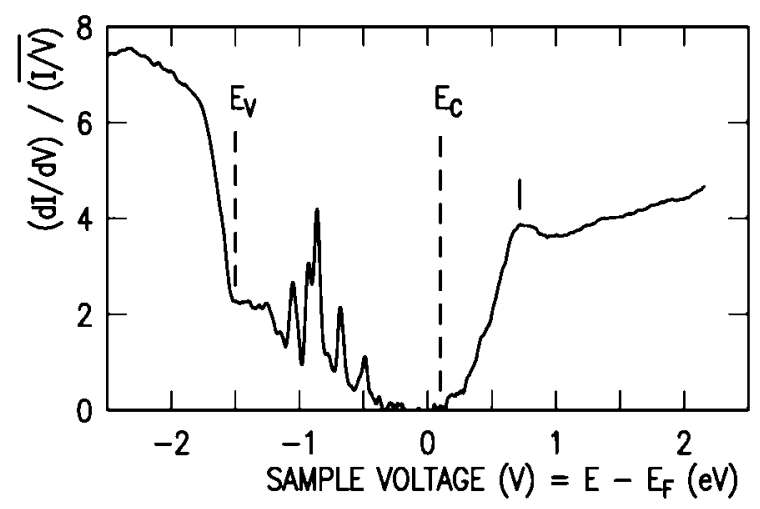

Fig. 2. Typical low-temperature tunneling spectrum (not near dopant atom) of $n$-type $\mathrm{GaAs}(110)$ surface, acquired at a temperature of $10 \mathrm{~K}$. The conduction band and valence band edges are indicated by dashed lines, and the ticmark at $+0.72 \mathrm{~V}$ indicates a surface state. The sharp lines in the bandgap region arise from localized accumulation layer states. For sample states probed by the tip Fermi level, the sample voltage corresponds to the energy of the state relative to the sample Fermi level.

LT and RT spectra for GaAs(110) appear quite similar, with the exception of the sharp spectral lines seen in the bandgap region for the LT spectrum. A nonzero current and conductance is also seen there for the RT spectrum, and this component of the current was correctly identified in early work as arising from an accumulation layer at the surface (forming the so-called "dopant induced" current component) [20]. In the case of low temperatures, discrete spectra lines arise in this region of the spectrum. These lines arise from the discrete localized states that form in the potential of the tip-semiconductor system; one can think of the potential beneath the tip as forming a type of "quantum dot" which travels through the semiconductor as the probe-tip moves [1]. As discussed below, the accumulation 
layers states are perturbed by the presence of a nearby dopant atom, and new lines associated with the dopant atom also appear in the spectra.

Figure 3 shows individual tunneling spectra acquired at the surface locations indicated in Fig. 1. (The spectra in this case are acquired over a narrower voltage range than that of Fig. 1, so that a constant sample-tip separation is used for the acquisition and we display simply the measured conductance with no normalization.) Spectra (a)-(e) were acquired on bare surface locations far from any defects or dopant atoms. The spectra all look similar, with a few (generally three) discrete lines observed in the range from -0.8 to $-1.2 \mathrm{~V}$. Occasionally the line nearest $0 \mathrm{~V}$ may have enhanced intensity (as in Fig. 3c), but we associate such a spectrum

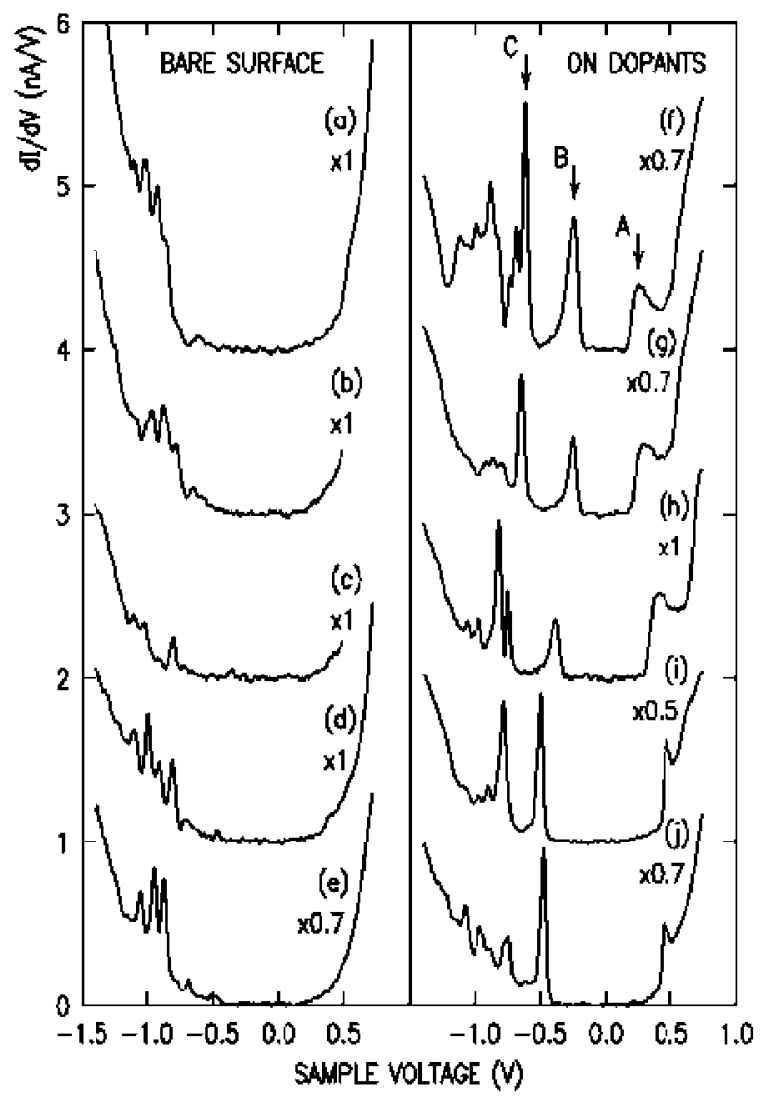

Fig. 3. Tunneling spectra acquired at the surface locations indicated in Fig. 1. Spectra (a)-(e) were acquired at locations far from dopant atoms, and spectra (f)-(j) were acquired directly on top of dopant atoms. Each spectrum is multiplied by the factor indicated prior to plotting, for ease of viewing. Successive spectra are shifted by one unit along the vertical axis. Discrete spectral lines observed at the dopant atoms are indicated by $A, B$, and $C$. 
with the unintentional proximity of a dopant or defect atoms. Spectra (f)-(j) were acquired at the location of dopant atoms. Three new spectral lines are visible in those spectra, labeled $A, B$, and $C$ in Fig. 3f. The first line, denoted $A$, occurs at positive sample voltages very near the onset of the $\mathrm{CB}$ component. The second line, denoted B, occurs at a negative voltage, whose magnitude is nearly equal to the voltage position of the $A$ line. The third line, denoted $C$, occurs at a negative voltage with magnitude slightly less than that of the accumulation layer spectral lines. In terms of its voltage position it is sometimes difficult to distinguish this line from those of the accumulation layer lines. However, we find that the line $C$ either displays a clear voltage separation from the accumulation layer lines or it displays a clear enhancement in intensity compared to the accumulation layer lines, so in all cases we regard it as a distinct, new spectral feature.

To identify the origin of the discrete spectral lines observed within the band gap region in the LT spectra of the $n$-type GaAs(110) surfaces, one must consider the tip-induced band bending in the semiconductor. We perform one-dimensional computations in a manner identical to that described in Ref. [20] [7]. Energy band profiles including semiconductor, vacuum, and probe-tip are pictured in Fig. 4 for a few choices of sample-tip voltages which turn out to be important in our discussion below. Those diagrams show the $\mathrm{CB}$ minimum of the sample together with the Fermi levels of tip and sample. Note that the diagrams are drawn to scale in the horizontal (distance) and vertical (energy) axes, as indicated in the figure.

Figure 4a shows the case of $0 \mathrm{~V}$ between sample and tip; we assume a tip work function of $4.5 \mathrm{eV}$ and GaAs electron affinity of $4.07 \mathrm{eV}$, so that depletion near the surface results, as seen in the diagram. The surface band bending is $0.18 \mathrm{eV}$. As the voltage is increased the semiconductor becomes more depleted, and a turn-on of the current occurs only when the tip Fermi level is aligned with the CB minimum at the surface, as pictured in Fig. $4 \mathrm{~b}$ (the sample-tip voltage required for this situation

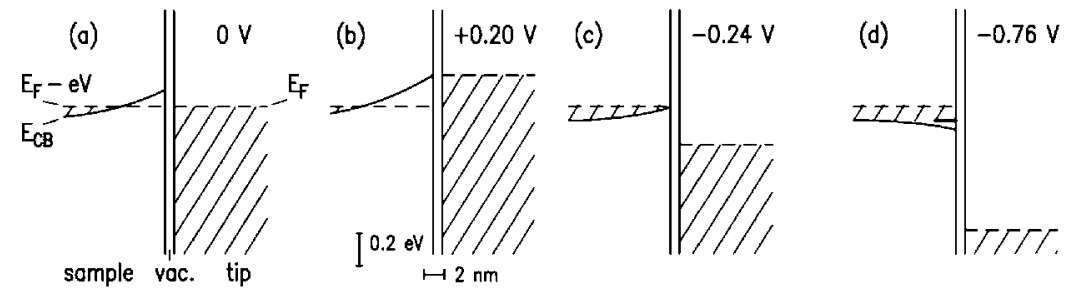

Fig. 4. Theoretical band profiles at energies near the Fermi levels of the metal probe-tip and the semiconductor sample. Profiles are drawn to scale in both energy and distance. $A$ sample-tip separation of $9 \AA$ is used. Case (a) shows the band profile for zero voltage between sample and tip. Cases (b)-(d) shows cases which correspond to the observed spectral lines $A-C$, respectively. The heavy line in case (d) indicates a localized accumulation layer state, just forming at that energy. 
is $+0.20 \mathrm{~V}$ ). Now, with a nearby dopant atom a discrete state is present just below the CB minimum, and this state will be thus seen in a spectrum near this voltage. We associate that state, i.e. tunneling into the dopant state, with the feature $A$ seen in our spectra. At negative sample-tip voltages, current from the occupied CB states into the tip will start to be observed, i.e. the dopant-induced component. This component turns on when the sample Fermi level is aligned with the CB minimum at the surface, as pictured in Fig. 4c (the sample-tip voltage required for that situation is $-0.24 \mathrm{~V}$ ). Again, with a nearby dopant atom a discrete line will be seen at this voltage, associated with tunneling out of the dopant atoms. We associate that feature with our observed spectral line B. Finally, at larger negative voltages localized states will form in the accumulation layer which exists at the surface. Using the WKB approximation we can make a non-self-consistent estimate of the voltages at the first localized states forms, which yields a voltage of $-0.76 \mathrm{eV}$, as shown in Fig. 4d. Near a dopant atom this state will be significantly perturbed. It will be localized laterally by the potential of the dopant atom, and vertically (into the semiconductor) by the band bending potential from the tip. A new type of state is formed, split off from the accumulation layer states, and we associate this state with the observed feature $C$ in our spectra.

The discrete spectral lines associated with the accumulation layer and dopant atoms, as seen in LT-STS spectra, have several applications. First, as mentioned in Sect. 1, the magnetic field dependence of the lines can provide detailed information on the physics of potential fluctuations and quantum states in the materials [2]. On a more applied basis, the position of the spectral lines can be used to determine the doping density in the semiconductor [7]. Essentially, the determination of doping density using tunnel current involves two main unknown parameters: the doping density itself and the contact potential between tip and sample. By observing two spectral features, the $A$ and $B$ lines, both of these unknowns can be determined. Referring to Fig. 4, and placing for ease of discussion the sample Fermi level right at the $\mathrm{CB}$ minimum, we see that the $B$ line position corresponds to the voltage required to obtain flat band conditions in the semiconductor. This voltage thus essentially equals the contact potential difference between tip and sample, thereby determining that parameter. Using the additional information provided by the position of the $A$ line, the doping density can be determined. We note that this technique for doping density determination is not restricted only to LT-STS measurements. Even at room temperature the dopant-induced (accumulation layer) component to the current is routinely observed [16]; the onset of that component is basically the same as the location of the $B$ line here, and the location of the $\mathrm{CB}$ component of the current is the same as the $A$ line position. With a series of band bending computations, RT or LT tunneling spectra of GaAs(110) surface can thus, in principle, be used to determine doping concentration of the GaAs. We do note, however, that a quantitative understanding of tip-induced band bending must also include consideration of the probe-tip curvature. The three-dimensional 
nature of the probe-tip can substantially reduce the tip-induced band bending [22], and those effects must also be included in any complete analysis of the problem.

$$
\text { 3.2. } G e(111) 2 \times 1 \text { and } G e(111) c(2 \times 8)
$$

Figure 5 shows a typical STM image acquired from our cleaved Ge(111) surfaces. As previously described, these surfaces consist of ordered $2 \times 1$ domains $(6.93 \times 4.00 \AA$ unit cell $)$, interspersed with areas containing disordered adatoms arrangements [23]. The surfaces generally contain about $50 \%$ of each type of structure. The size of the domains varies from cleave to cleave, but typically it is about $5 \mathrm{~nm}$. Tunneling spectra were acquired from the center of well-ordered $2 \times 1$ domains.

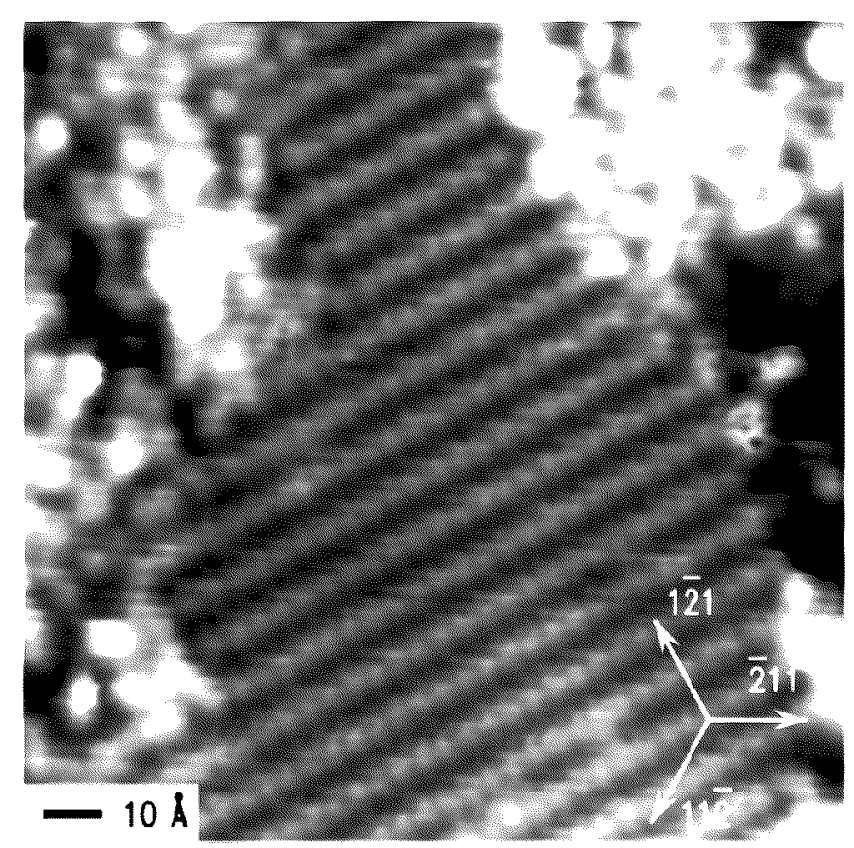

Fig. 5. STM image of cleaved Ge(111) surface. A well-ordered $2 \times 1$ domain is seen in the center of the image, with disordered adatom arrangement visible on the right and left sides of the image. The image was acquired with a sample voltage of $-1.5 \mathrm{~V}$ and constant tunnel current of $1 \mathrm{nA}$. Gray scale range is $0.9 \AA$.

Figure 6 presents results for a tunneling spectrum obtained from the Ge(111) $2 \times 1$ surface. This LT spectrum displays features very similar to prior RT spectra [23], except that the peaks are generally narrower and 2-3 times more intense in the LT case. Spectral features can be located in Fig. 6 with a precision of typically $\pm 0.02 \mathrm{eV}$. The bottom of the empty surface state band appears as the intense peak at $0.19 \mathrm{eV}$ (all energies relative to the Fermi level, $0 \mathrm{~V}$ ). A 


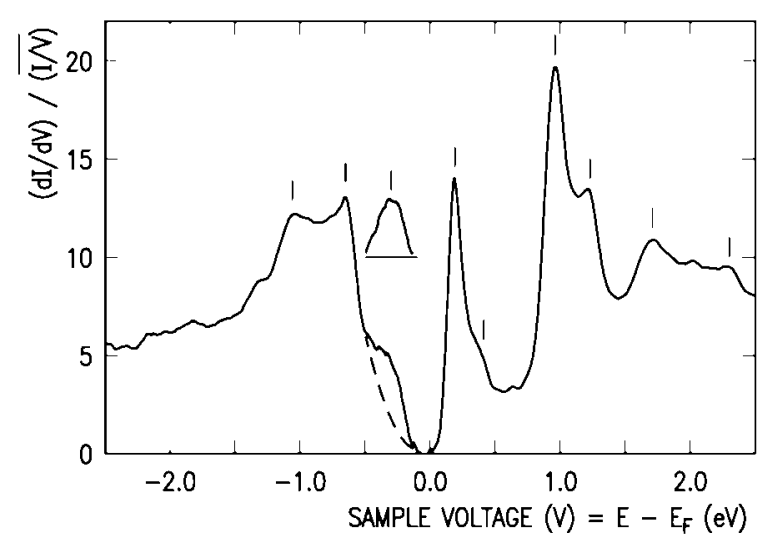

Fig. 6. Low-temperature tunneling spectrum $(12 \mathrm{~K})$ of the Ge(111)2 $\times 1$ surface. Peak positions are indicated by vertical lines. The dashed line is an approximate background used for determining the peak position of the feature near $-0.3 \mathrm{~V}$, with the difference spectrum shown above raw spectrum. The sample voltage corresponds to the energy of a. state relative to the Fermi level $(0 \mathrm{~V})$.

small shoulder is reproducibly seen on the high energy side of this peak, at about $0.41 \mathrm{eV}$. The upper part of this band appears as two peaks (only one peak was seen at room temperature), at 0.96 and $1.23 \mathrm{eV}$. Higher energy peaks, associated with bulk bands, are seen at 1.71 and $2.30 \mathrm{eV}$. The top of the filled state band is seen as a relatively weak shoulder (similar to the room temperature results), probably because of mixing with valence band states. Using an approximate background subtraction shown by the dashed line in Fig. 6, we deduce an energy location of $-0.30 \mathrm{eV}$ with uncertainty of $\pm 0.04 \mathrm{eV}$. Two peaks are seen associated with the lower portions of the filled state band, at -0.65 and $-1.06 \mathrm{eV}$. The remaining small features in the spectrum are at the limit of the residual vibrational noise in our STM, and cannot be reliably identified.

A significant advantage to LT surface state spectra such as that shown in Fig. 6, compared to the RT results, is the increased spectral resolution arising from a reduction in thermal broadening. Another aspect of the reduced temperature is that the transport mechanisms for the carriers in the semiconductor may be modified. In most STS results it is assumed that the limiting factor in the transport is the vacuum tunneling event itself. Nevertheless, several recent works indicate that novel results can be obtained at reduced temperatures [9, 10, 12], or even at room temperature when the magnitude of the tunnel current is varied [24]. Results of this type are shown below for the $\operatorname{Ge}(111) \mathrm{c}(2 \times 8)$ surfaces [13].

As mentioned in Sect. 2, the $c(2 \times 8)$ surfaces were formed by annealing the as-cleaved $\mathrm{Ge}(111)$ surfaces. Large domains of well-ordered $c(2 \times 8)$ are formed by this procedure [25]. Spectra acquired from the $c(2 \times 8)$ surfaces are shown in Fig. 7 , 


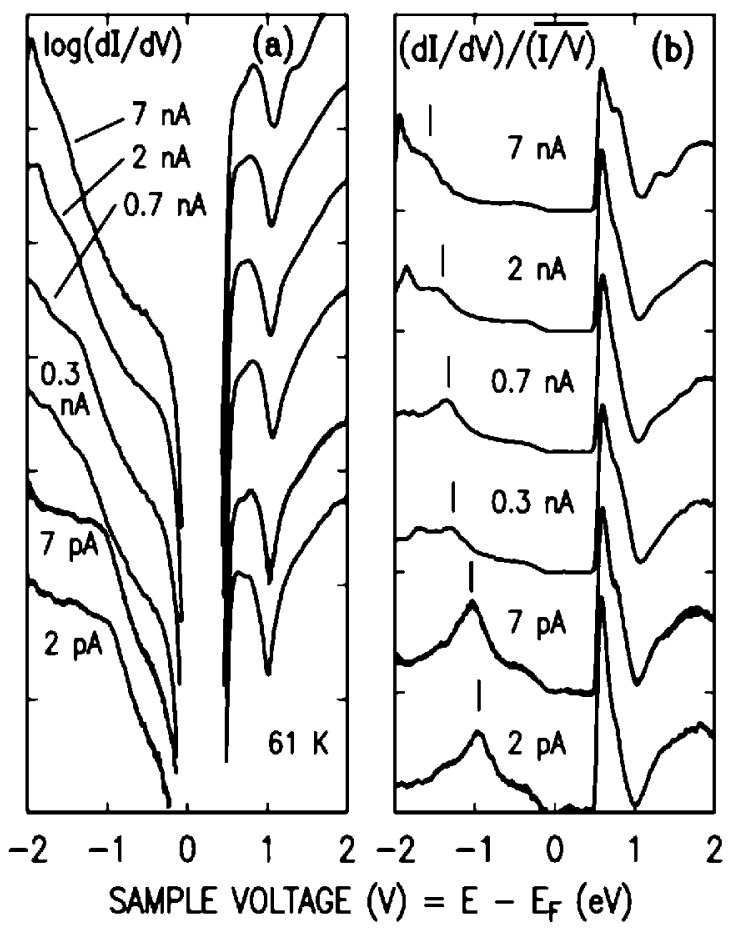

Fig. 7. Tunneling spectra obtained from the $\mathrm{Ge}(111) \mathrm{c}(2 \times 8)$ surface at $61 \mathrm{~K}$ and various current setpoints. Spectra are shown as (a) $\mathrm{d} I / \mathrm{d} V$ at constant tip-sample separation on a logarithmic scale (one order-of-magnitude per division) and (b) normalized conductance on a linear scale. The spectra at different setpoints are shifted vertically, for clarity. The rest-atom spectral peak is indicated by the ticmarks in (b).

acquired in this case at a temperature of $61 \mathrm{~K}$. Spectra are shown for different tunnel current setpoints, corresponding to the current at a voltage near one of the endpoints of the spectra. The current setpoint thus determines the tip-sample separation. The $\mathrm{Ge}(111) \mathrm{c}(2 \times 8)$ surface is known to consist of an arrangement of Ge adatoms and rest-atoms [26]. The large spectral peak seen in Fig. 7 centered at about $+0.7 \mathrm{~V}$ is derived from the adatoms, and the smaller peak at about $-1.2 \mathrm{~V}$ is derived from the rest-atoms. Remarkably, the rest-atom peak is seen to shift continuously as a function of the current setpoint. One possible mechanism for this shift is the tip-induced band bending discussed above for the GaAs(110) surface; as the tunnel current setpoint increases the tip-sample separation decreases and hence tip-induced band bending will increase. We have performed detailed computations of this effect (for zero tunnel current), using the full three-dimensional tip-sample geometry. We find however that the rate of change of the tip-induced band bending effect with tip-sample separation is a factor of 4-5 slower than that deduced from the spectra of Fig. 7 [13]. We thus attribute the observed shifts in the rest-atom 
peak to an accumulation of tunneling carriers in the near-surface region of the semiconductor, in agreement with the prior work of Dujardin et al. [12]. The charge density of these nonequilibrium carriers will produce additional changes to the electrostatic potential profile, in particular producing additional band bending, which is also found to be temperature dependent $[12,13]$.

\section{Summary}

In summary we have presented several examples of low-temperature STS studies, for $\operatorname{GaAs}(110), \mathrm{Ge}(111) 2 \times 1$, and $\mathrm{Ge}(111) \mathrm{c}(2 \times 8)$ surfaces. In all cases the effect of reducing the temperature is to enable a great increase in spectral resolution. For $\operatorname{GaAs}(110)$, a surface for which no surface states exist in the bandgap region, this increased resolution permits the observation of discrete lines associated with accumulation layers states. Additional lines are observed near dopant atoms, and it is argued that the position of these lines can be used to deduce the doping concentration of the material. For Ge(111) $2 \times 1$, the spectrum is dominated by surface states which extend throughout the bandgap region. The reduced temperatures again lead to significant sharpening of the spectral features, permitting a better identification of their origins. For $\mathrm{Ge}(111) \mathrm{c}(2 \times 8)$, the spectral results are found to depend on both temperature and tunnel current magnitude, indicating some limitations in the transport of carriers in the semiconductor. The ability to vary temperature (and tunnel current) is thus found to be an important parameter for probing this nanoscale transport phenomenon in the semiconductor.

\section{Acknowledgments}

The work has been supported by the A. von Humboldt Foundation and by the U.S. National Science Foundation.

\section{References}

[1] R. Dombrowski, Chr. Steinebach, Chr. Wittneven, M. Morgenstern, R. Wiesendanger, Phys. Rev. B 59, 8043 (1999).

[2] M. Morgenstern, Chr. Wittneven, R. Dombrowski, R. Wiesendanger, Phys. Rev. Lett. 84, 5588 (2000).

[3] M.C.M.M. van der Wielen, A.J.A. van Roij, H. van Kempen, Phys. Rev. Lett. 76, 1075 (1996).

[4] Chr. Wittneven, R. Dombrowski, M. Morgenstern, R. Wiesendanger, Phys. Rev. Lett. 81, 5616 (1998).

[5] A. Depuydt, C. Van Haesendonck, N.S. Maslova, V.I. Panov, S.V. Savinov, P.I. Arseev, Phys. Rev. B 60, 2619 (1999).

[6] R. de Kort, M. C.M.M. van der Wielen, A.J.A. van Roij, W. Kets, H. van Kempen, Phys. Rev. B 63, 125336 (2001). 
[7] R.M. Feenstra, G. Meyer, F. Moresco, K.H. Rieder, Phys. Rev. B 66, 165204 (2002).

[8] S.Y. Tong, A.R. Lubinsky, B.J. Mrstik, M.A. Van Hove, Phys. Rev. B 17, 3303 (1978)

[9] T. Yokoyama, K. Takayanagi, Phys. Rev. B 61, 5078 (2000).

[10] T. Mitsui, K. Takayanagi, Phys. Rev. B 62, 16251 (2000).

[11] R.M. Feenstra, G. Meyer, F. Moresco, K.H. Rieder, Phys. Rev. B 64, 081306 (2001).

[12] G. Dujardin, A.J. Mayne, F. Rose, Phys. Rev. Lett. 89, 036802 (2002).

[13] R.M. Feenstra, G. Meyer, K.H. Rieder, to be published.

[14] G. Meyer, Rev. Sci. Instrum. 67, 2960 (1996).

[15] P. Mårtensson, R.M. Feenstra, Phys. Rev. B 39, 7744 (1989).

[16] R.M. Feenstra, Phys. Rev. B 50, 4561 (1994).

[17] M.B. Johnson, O. Albrektsen, R.M. Feenstra, H.W.M. Salemink, Appl. Phys. Lett. 63, 2923 (1993); 64, 1454 (1994).

[18] J.F. Zheng, X. Liu, N. Newman, E.R. Weber, D.F. Ogletree, M. Salmeron, Phys. Rev. Lett. 72, 1490 (1994).

[19] C. Domke, Ph. Ebert, M. Heinrich, K. Urban, Phys. Rev. B 54, 10288 (1996).

[20] R.M. Feenstra, J.A. Stroscio, J. Vac. Sci. Technol. B 5, 923 (1987).

[21] R.M. Feenstra, Phys. Rev. B 50, 4561 (1994).

[22] R.M. Feenstra, J. Vac. Sci. Technol. B 21, 2080 (2003).

[23] R.M. Feenstra, Phys. Rev. B 44, 13791 (1991).

[24] V. Ramachandran, R.M. Feenstra, Phys. Rev. Lett. 82, 1000 (1999).

[25] R.M. Feenstra, A.J. Slavin, Surf. Sci. 251/252, 401 (1991).

[26] R.S. Becker, B.S. Swartzentruber, J.S. Vickers, T. Klistner, Phys. Rev. B 39, 1633 (1989). 